\title{
The Differential Diagnosis of Colorectal Polyps Using Colon Capsule Endoscopy
}

\author{
Kei Nakazawa ${ }^{1,2}$, Sadaharu Nouda ${ }^{1}$, Kazuki Kakimoto ${ }^{1}$, Naohiko Kinoshita ${ }^{1}$, \\ Yasuyoshi Tanaka ${ }^{1}$, Hideki Tawa ${ }^{1}$, Ryoji Koshiba ${ }^{1}$, Yutaka Naka ${ }^{1}$, Yuki Hirata ${ }^{1}$, \\ Kazuhiro Ota ${ }^{1}$, Ken Kawakami ${ }^{1}$, Toshihisa Takeuchi ${ }^{1}$, Takuya Inoue ${ }^{1}$, Takako Miyazaki ${ }^{1}$, \\ Makoto Sanomura $^{3}$, Shiro Nakamura ${ }^{1}$, Yutaka Saito ${ }^{2}$ and Kazuhide Higuchi ${ }^{1}$
}

\begin{abstract}
:
Objective Although colorectal polyps (CPs) can be observed with colon capsule endoscopy (CCE), it is difficult to determine the type of polyp using CCE. The objective of this study was to differentiate adenomatous polyps (APs) from hyperplastic polyps (HPs) with CCE.

Methods In this single-center retrospective study, an analysis was conducted on the same CPs with both CCE and colonoscopy (CS) and histopathologically diagnosed as AP or HP. The color difference ( $\triangle \mathrm{E})$ between the polyp surface and the surrounding mucosa was calculated using the CIE1976 $L^{*} a^{*} b^{*}$ color space method on white light (WL), flexible spectral imaging color enhancement (FICE), and blue mode (BM) CP images. We investigated the ability of the ratio of the color differences $\left(\Delta \mathrm{E}^{\prime}\right)$ to differentiate between APs and HPs.

Results The size of all 51 polyps (34 APs, $17 \mathrm{HPs}$ ) was $7.5 \pm 4.6 \mathrm{~mm}$ with CCE and $7.3 \pm 4.2 \mathrm{~mm}$ with CS, and this difference was not significant $(\mathrm{p}=0.28)$. The FICE $\Delta \mathrm{E}^{\prime}$ of APs was $3.3 \pm 1.8$, which was significantly higher than the FICE $\Delta \mathrm{E}$ ' of HPs $(1.3 \pm 0.6 ; \mathrm{p}<0.001)$. A receiver operating characteristic analysis showed that FICE $\triangle \mathrm{E}$ ' was useful for differentiating between APs and HPs, with an area under the curve of 0.928 (95\% confidence interval, $0.843-1$ ). The sensitivity was $91.2 \%$, and the specificity was $88.2 \%$ with a cut-off value of 1.758 .

Conclusion Using FICE on CCE images of CPs and applying the CIELAB color space method, we were able to differentiate between APs and HPs with high accuracy. This method has the potential to reduce unnecessary CS procedures.
\end{abstract}

Key words: colon capsule endoscopy, colorectal polyp, adenomatous polyp, hyperplastic polyp, flexible spectral imaging color enhancement

(Intern Med 60: 1805-1812, 2021)

(DOI: 10.2169/internalmedicine.6446-20)

\section{Introduction}

The incidence of colorectal cancer is increasing worldwide $(1,2)$; however, secondary prevention by the early detection and treatment of colorectal polyps (CPs) provides the possibility of a complete cure (3-5). Colonoscopy (CS) is a very effective method for identifying and removing CPs.
However, it is an invasive procedure that can cause pain in some patients, and the complications associated with CS may include perforation and bleeding. The first generation of colon capsule endoscopy (CCE) (PillCam Colon; Given Imaging, Yoqneam, Israel) procedures appeared in 2006, offering a pain-free, noninvasive examination (6). Technological advances led to a second generation of CCE in 2009; this technology is reportedly associated with high CP detec-

${ }^{1}$ 2nd Department of Internal Medicine, Osaka Medical College, Japan, ${ }^{2}$ Endoscopy Division, National Cancer Center Hospital, Japan and ${ }^{3}$ Department of Gastroenterology, Hokusetsu General Hospital, Japan

Received: October 6, 2020; Accepted: November 11, 2020; Advance Publication by J-STAGE: January 15, 2021

Correspondence to Dr. Kazuki Kakimoto, in2098@osaka-med.ac.jp 
tion rates of approximately $84-94 \%$ with polyps $\geq 6 \mathrm{~mm}$ and $88-92 \%$ with polyps $\geq 10 \mathrm{~mm}$ (7-9).

If a CP detected with CCE is identified as a neoplastic lesion (NL), CS should be performed to remove it. However, if the polyp is a non-neoplastic lesion $(\mathrm{NN})$, it will not require endoscopic resection (ER), and unnecessary CS should be avoided. Thus far, it has not been possible to differentiate between NLs and NNs with CCE $(10,11)$. Therefore, CS is generally performed even for NNs that do not require ER.

Second-generation CCE is equipped with flexible spectral imaging color enhancement (FICE) and blue mode (BM), which are digital image processing technologies that use the RAPID software program (Given Imaging, Yoqneam, Israel, V.7.0) (12). FICE creates a new and flexible spectral image by approximating spectral reflectance from the white light (WL) image of an object and selecting and reconstructing red, green, and blue (RGB) wavelengths that emphasize the object (13-15). The BM superimposes the color coefficient shift of light in the short-wavelength range $(490-430 \mathrm{~nm})$ on a WL image (16). Imagawa et al. reported that using FICE in small bowel capsule endoscopy (SBCE) achieved greater visibility of angioectasia, erosion/ulceration, and tumors than WL (17). Similarly, Sato et al. reported that the detection rate of small bowel lesions increased when using FICE (18). Improved visibility of blood vessels and the demarcation of lesion borders has been reported when using BM in SBCE (19). However, there have been no reports on whether or not CCE can differentiate between different types of polyps.

The present study investigated whether or not the CIE $1976 L^{*} a * b^{*}$ (CIELAB) color space method $(20,21)$ could be used to differentiate between adenomatous polyps (APs) and hyperplastic polyps (HPs) in WL, FICE, and BM images of CPs obtained using CCE.

\section{Materials and Methods}

\section{Patients}

This was a retrospective single-center study. From April 2014 to December 2018, we recruited consecutive patients in whom CCE identified a CP of $\geq 6 \mathrm{~mm}$ and who underwent CS for ER within 1 month after CCE. The Ethics Committee of Osaka Medical College approved this study.

\section{Study protocol}

The analysis was performed on lesions that were identified as CCE and confirmed to be the same polyps resected during CS and that had been histopathologically diagnosed as AP or HP by ER or a biopsy. The size and morphology of each polyp in CS and CCE images were compared. In addition, the color difference between the polyp surface and the surrounding mucosa was calculated from WL, FICE, and $\mathrm{BM}$ images using the CIELAB color space method to determine whether or not this approach could be used to differentiate between APs and HPs.
If the following criteria were met, the lesion was judged to be the same in CCE and CS: 1) the polyps diagnosed with CCE and CS were in the same or adjacent segments (with the large intestine divided into four segments: cecum to ascending colon, transverse colon, descending colon to sigmoid colon, and rectum); 2) the error in the size of the polyps evaluated by CCE and CS was $\pm 50 \%$ or less; and 3 ) the interpreter determined that the lesion was the same polyp identified with CCE and CS.

\section{CCE and CS procedures}

The examinations were performed via second-generation CCE (PillCam ${ }^{\circledR}$ COLON2; Covidien Japan, Tokyo, Japan). The CCE pretreatment protocol followed that of a previous report (9). The CCE system consists of a capsule, a data recorder (DR3), and a computer equipped with the RAPID software program (9). The CCE system has cameras with a viewing angle of $172^{\circ}$ at both ends, enabling the acquisition of almost $360^{\circ}$ images. It is also able to perform polyp size estimation (PSE), which can measure the size of the lesion (22). Thus, the sizes of the CPs that were found were measured using PSE. The location of the lesion was estimated based on the landmarks captured by CCE (cecum, hepatic flexure, splenic flexure, and rectum) and on the trajectory of the capsule, as displayed in the RAPID software program (23). The macroscopic type of CPs was divided into protruded and superficial types using the Paris endoscopic classification (24). The CCE diagnosis was performed by two practitioners $(\mathrm{KN}, \mathrm{SN})$, each with experience in at least 20 cases.

A PCF-H290I or PCF-Q260AZI (Olympus, Tokyo, Japan) was used for the CS procedure. The CP observed with CCE was carefully identified with CS to perform ER (cold snare polypectomy or endoscopic mucosal resection) or a biopsy. With CS, the size of the CP was measured using biopsy forceps or a ruler. CS was performed by 4 practitioners (KN, $\mathrm{SN}, \mathrm{YH}, \mathrm{KK}$ ), including the 2 practitioners (KN, SN) who performed $\mathrm{CCE}$ diagnosis, each with experience in at least 1,000 cases.

\section{Calculation of the color differences}

The CIELAB color space is the most widely used method for measuring and ordering object color $(20,21)$. The color coordinates are: $L^{*}$ for the lightness from black (0) to white (100), $a^{*}$ from green (-) to red (+), and $b^{*}$ from blue $(-)$ to yellow (+) (Fig. 1). In this study, the color difference between the polyp surface (p) and the normal mucosa (b) in the CCE image of a CP was calculated for WL, FICE, and BM. The normal mucosa was detected from the mucosa surrounding the polyp and did not include residues, bubbles, or blood vessels in WL images. The color difference was the distance between two points in the $L^{*} a^{*} b^{*}$ color space calculated using the formula

$$
\Delta \mathrm{E}_{\mathrm{pb}}=\sqrt{\left(L^{p}-L^{b}\right)^{2}+\left(a^{p}-a^{b}\right)^{2}+\left(b^{p}-b^{b}\right)^{2}}
$$


In brief, 1) corresponding regions (polyp surface and surrounding mucosa, 64 pixels each) were selected in the Adobe Photoshop Elements 15 software program (Adobe Systems, San Jose, USA) (Fig. 2); 2) the median RGB value was then determined; 3) the $L * a * b *$ value was calculated from the mean RGB value; and 4) the $L^{*} a * b *$ value was used to calculate $\Delta \mathrm{E}$. We examined the color differences in the CCE images of AP and HP separately (Fig. 3). Furthermore, the ratio of the color differences in the FICE and WL images was defined as FICE $\Delta \mathrm{E} / \mathrm{WL} \Delta \mathrm{E}$ (FICE $\Delta \mathrm{E}$ ').

The utility of the FICE $\Delta \mathrm{E}$ ' value to differentiate between APs and HPs was then examined via a receiver operating characteristic (ROC) analysis.

\section{Statistical analyses}

Wilcoxon's signed-rank test was used to compare $\mathrm{CP}$ sizes. The paired $t$-test was used to compare $\Delta \mathrm{E}$ and $\Delta \mathrm{E}^{\prime}$.

\section{The CIELAB color space}

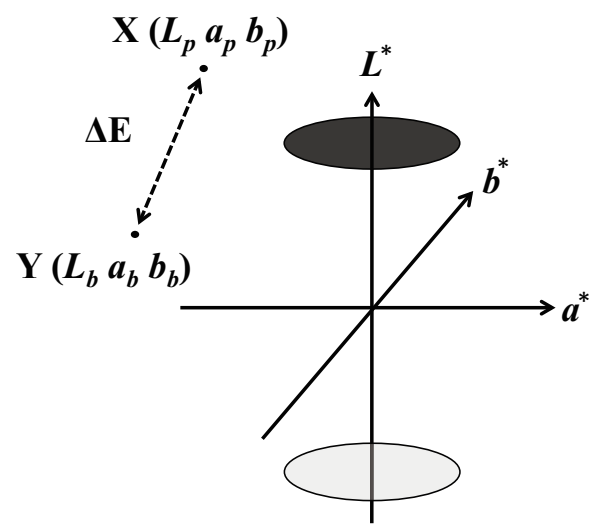

Figure 1. CIE $1976 L^{*} a * b *$ (CIELAB) color space. The color coordinates are: $L^{*}$ for the lightness from black $(0)$ to white (100), $a^{*}$ from green (-) to red (+), and $b^{*}$ from blue (-) to yellow $(+) .(X)$ is the polyp surface; $(Y)$ is the surrounding mucosa. The color difference $(\Delta \mathrm{E})$ was the distance between two points calculated using the formula $\Delta \mathrm{E}_{\mathrm{pb}}=\sqrt{\left(L^{p}-L^{b}\right)^{2}+\left(a^{p}-a^{b}\right)^{2}+\left(b^{p}-b^{b}\right)^{2}}$.
Fisher's exact test was used to compare the morphological diagnoses with $\mathrm{CS}$ and CCE. The statistical significance level was set as $\mathrm{p}<0.05$.

All statistical analyses were performed using EZR (Saitama Medical Center, Jichi Medical University, Saitama, Japan).

\section{Results}

Of the 49 patients who underwent CCE at our hospital, polyps $\geq 6 \mathrm{~mm}$ in size were observed in 19 . Of these, 18 patients underwent CS for therapeutic purposes. From these examinations, 53 lesions were judged to be the same as those identified with CCE. The 18 patients included 11 men and 7 women with a median age of $71(35-87)$ years old (Table 1). The histopathological diagnoses were 34 cases of AP, 17 of HP, 1 sessile serrated adenoma/polyp (SSA/P), and 1 traditional serrated adenoma (TSA). We analyzed 51 lesions, excluding SSA/P and TSA. The polyps were located from the cecum to the ascending colon in 12 cases, in the transverse colon in 10 cases, from the descending to the sigmoid colon in 24 cases, and in the rectum in 5 cases by CS.

The overall polyp size was $7.5 \pm 4.6 \mathrm{~mm}$ on CCE and 7.3 \pm $4.2 \mathrm{~mm}$ on CS, and this difference was not significant (Table 2). There was no marked difference between the CCE and CS measurements when the polyps were separated into APs and HPs. Macroscopically, CPs were identified as protruded in 42 cases and superficial in 9 cases with CCE and as protruded in 39 cases and superficial in 12 cases with CS (including lateral spreading tumors; LSTs), with a diagnostic agreement of $92.2 \%$ (47/51 cases). Of the discrepancies, three cases were diagnosed as protruded on CCE but superficial on CS, and one case was diagnosed as superficial on CCE but protruded on CS.

\section{The comparison of color differences in CCE images}

Among the 34 APs, the FICE $\Delta E(39.2 \pm 19.3)$ was significantly higher than the WL $\Delta \mathrm{E}(14.9 \pm 10.4)(\mathrm{p}<0.001)$, as was the $\mathrm{BM} \Delta \mathrm{E} \quad(23.3 \pm 15.4) \quad(\mathrm{p}<0.001) \quad$ (Fig. 4). In contrast,
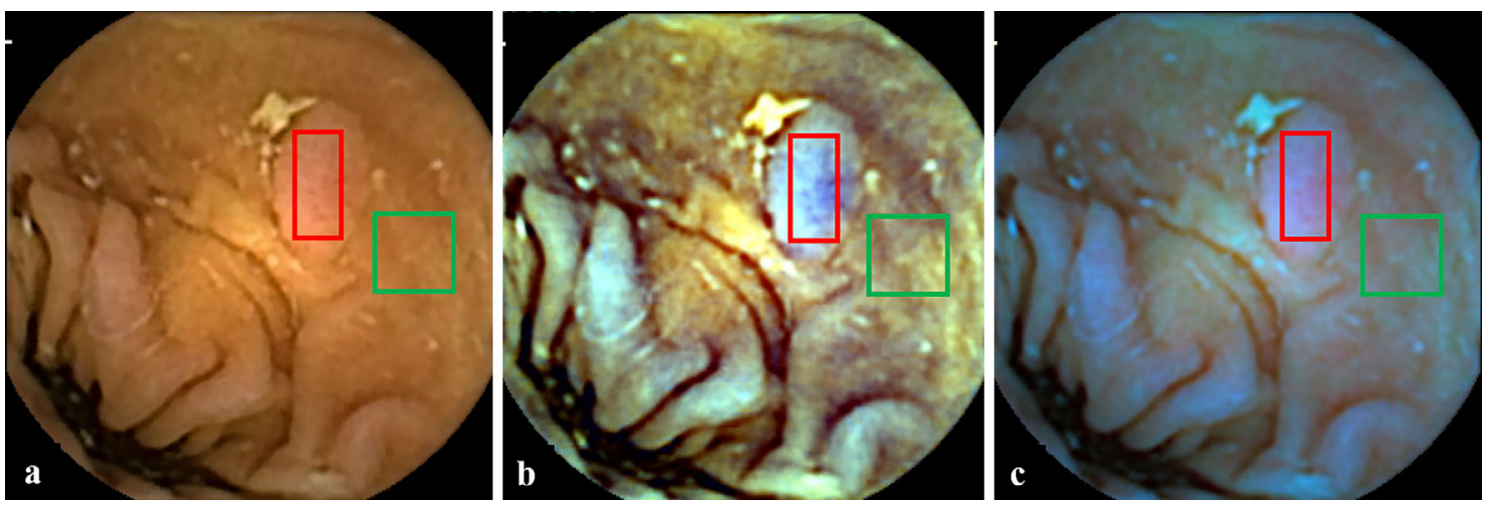

Figure 2. Selection of points for assessing the color differences in colon capsule endoscopy (CCE) images. Red square areas consisting of $64(8 \times 8)$ pixels show the polyp surface. Green square areas show the surrounding mucosa. (a) White light (WL), (b) flexible spectral imaging color enhancement (FICE), and (c) blue mode (BM). 


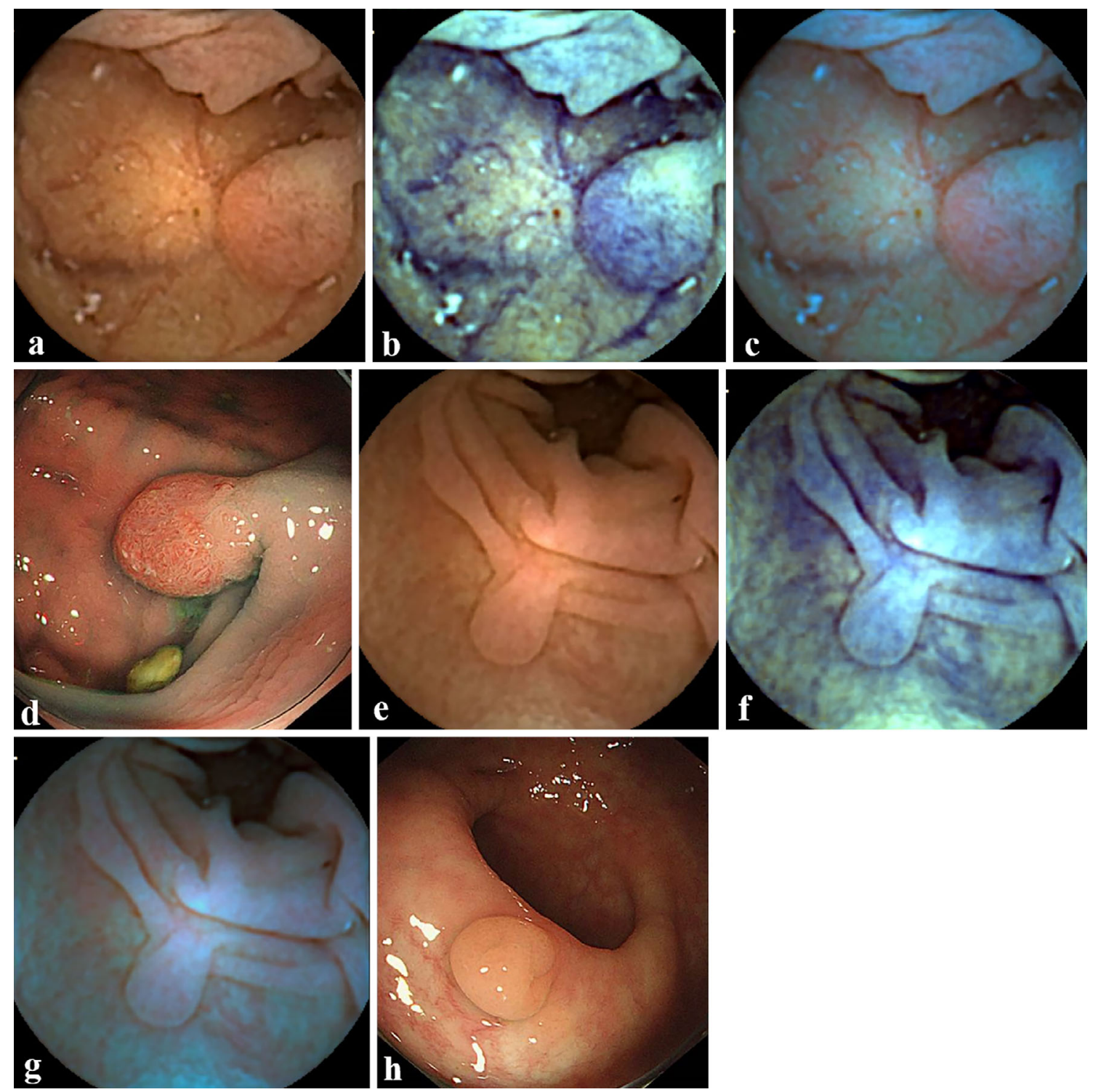

Figure 3. WL, FICE, and BM colon polyp images with colon capsule endoscopy (CCE) and WL images with CS. (a-d) The same adenomatous polyp, (e-h) the same hyperplastic polyp. (a, e) CCE WL images, (b, f) CCE FICE images, (c, g) CCE BM images, and (d, h) CS WL images.

among the $17 \mathrm{HPs}$, the FICE $\Delta \mathrm{E}(12.8 \pm 10.4)$ and $\mathrm{BM} \Delta \mathrm{E}$ $(13.1 \pm 11.9)$ were not significantly different from the WL $\Delta \mathrm{E}$ $(10.7 \pm 6.8)(\mathrm{p}=0.44$ and $\mathrm{p}=0.45$, respectively) (Fig. 4).

\section{Differentiation of adenoma and hyperplastic polyps using $\Delta E^{\prime}$}

We attempted to differentiate APs and HPs using $\Delta \mathrm{E}^{\prime}$ from the CCE images. The FICE $\Delta \mathrm{E}$ ' of the APs $(3.3 \pm 1.8)$ was significantly higher than that of the HPs $(1.3 \pm 0.6$; $\mathrm{p}<$

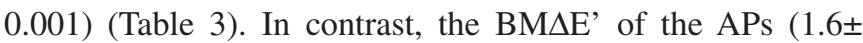
$0.5)$ was not significantly different from that of the HPs $(1.2$ \pm 0.6 ; $=0.23$ ).

In the ROC analysis, the FICE $\triangle \mathrm{E}$ ' was shown to be useful for differentiating between APs and HPs, with an area under the curve of 0.928 (95\% confidence interval, 0.843-1) (Fig. 5). The optimal cut-off value for differentiation was 1.758. This cut-off value had $91.2 \%$ sensitivity and $88.2 \%$ specificity.

Influence of the factors relevant to anemia on the calculation of the FICE $\Delta E^{\prime}$

Of the 18 patients, 3 had mild anemia and had 8 polyps. Fifteen patients without anemia had 43 polyps. The FICE $\Delta E$ ' of patients with anemia was $3.1 \pm 1.0$, which was not significantly different from the FICE $\Delta E$ ' of patients without anemia $(2.5 \pm 1.9 ; \mathrm{p}=0.195)$. Regarding gender differences, the FICE $\Delta \mathrm{E}$ ' of the 11 men was $2.7 \pm 2.0$, which was not significantly different from the FICE $\Delta E$ ' of the 7 women $(2.5 \pm 1.5 ; \mathrm{p}=0.756)$.

\section{Discussion}

This study demonstrated that APs and HPs can be differentiated with high accuracy using FICE on CCE to perform 
Table 1. Baseline Characteristics of the Patients and Polyps.

\begin{tabular}{lc}
\hline \multicolumn{1}{c}{ Characteristic } & Value \\
\hline Number of patients & 18 \\
Sex (male/female) & $11 / 7$ \\
Age, years & $71(63-79)$ \\
Number of polyps & 51 \\
Polyp location & $12: 10: 24: 5$ \\
(Cecum-A/C:T/C:D/C-S/C:rectum) & $34: 17(66.7: 33.3)$ \\
Pathological diagnosis & \\
(Adenomatous polyp, hyperplastic polyp), $\mathrm{n}(\%)$ & \\
\hline
\end{tabular}

A/C: ascending colon, T/C: transverse colon, D/C: descending colon, S/C: sigmoid colon

Table 2. Size and Macroscopic Type of Polyps with CCE and CS.

\begin{tabular}{lccc}
\hline & CCE & CS & p value \\
\hline Size (mm) & & & \\
overall & $7.5 \pm 4.6$ & $7.3 \pm 4.2$ & 0.275 \\
Adenomatous polyps & $8.5 \pm 4.6$ & $8.1 \pm 4.1$ & 0.135 \\
Hyperplastic polyps & $5.5 \pm 4.0$ & $5.6 \pm 3.8$ & 0.986 \\
Macroscopic type & $42: 9$ & $39: 12$ & 0.292 \\
(Protruded:Superficial) & & & \\
\hline
\end{tabular}

CCE: colon capsule endoscopy, CS: colonoscopy

a CIELAB color difference analysis. This study is the first to differentiate between APs and HPs on CCE. As it is not possible to determine the type of polyp with CCE, there is no choice but to perform CS when a CP is identified (11). However, although there is a risk of malignancy with APs due to the adenoma-carcinoma sequence $(25,26)$, HPs are unlikely to become malignant (27). Therefore, if a polyp observed using CCE is shown to be an HP, CS is generally unnecessary unless the polyp is relatively large. In the present study, good differentiation was made possible by calculating the FICE $\Delta \mathrm{E}$ ' from CCE images, with a sensitivity of $91.2 \%$ and specificity of $88.2 \%$. This method may be of great clinical significance for reducing unnecessary CS procedures.

A diagnosis using CCE should first be performed conventionally with WL images. With CCE, the most important thing is not to miss colorectal cancer. If a lesion is suspected of being colorectal cancer, CS must be performed, regardless of the size of the lesion. Differentiation using color differences is at most an auxiliary diagnosis, and its significance lies in identifying cases where CS is not necessary. The present study examined APs and HPs and did not include colorectal cancer. Because colorectal cancer appears redder than APs (28), we expect the FICE $\Delta \mathrm{E}$ ' of colorectal cancer to be equal to or greater than that of APs. NLs that are $\geq 6 \mathrm{~mm}$ are more often cancerous than those that are $<6$ $\mathrm{mm}$, and ER is recommended (29-31). Although the frequency of cancer with lesions $<6 \mathrm{~mm}$ is extremely low, at $0.03-0.3 \%(31,32)$, ER may be performed to prevent progression to cancer, depending on the patient's age, overall condition, and comorbidities $(33,34)$. HPs, which are NNs, are flat, white elevations frequently found in the rectum and sigmoid colon. Lesions with a diameter $<6 \mathrm{~mm}$ are unlikely to become tumors in the future, and the recommendation is to watch and wait (33-35). Recently, SSA/Ps have attracted attention because they are thought to be precursors of MSIpositive colorectal cancer (36). SSA/Ps occur predominantly in the right colon and have a malignant transformation rate of $3.0-15 \%(37,38)$. Lesions $\geq 10 \mathrm{~mm}$ are indicated for ER (39-41). Because SSA/Ps are flat and have a faded color; they can be difficult to differentiate from HPs using endoscopic findings with WL CS (42). While we did not examine any such lesions, they may be difficult to differentiate from HPs even when using the FICE $\Delta \mathrm{E}$ ' determined from CCE. Therefore, a comprehensive assessment that includes several factors, such as the location and size, must be performed to determine whether or not therapy is indicated.

For all of these reasons, if a lesion is $<6 \mathrm{~mm}$ and the FICE $\Delta \mathrm{E}^{\prime} \leq 1.76$ and the location in the rectum or sigmoid colon, it can be considered HP, and a watch-and-wait approach can be applied. If a lesion is 6-9 $\mathrm{mm}$ and the FICE $\Delta \mathrm{E}^{\prime} \leq 1.76$, CS may not be required immediately, as the lesion may be an HP. However, the patient should undergo CCE or CS after one year or more.

Few reports have compared the macroscopic type of polyps with CCE and CS (9). Although the macroscopic type matched in most cases in the present study, there was disagreement in $7.8 \%$ (4/51) of cases. Three cases were diagnosed as protruded with CCE but as superficial with CS. This may be because observations using CCE are performed while immersed in fluid, and if the intestinal tract is insufficiently dilated, lesions may be more likely to appear protruded. Furthermore, one lesion was diagnosed as superficial with CCE but as protruded with CS. In this case, the polyp was not observed entirely on CCE, which made it difficult to assess its elevation. General differences in the observation conditions of CCE and CS may have led to differences in the macroscopic type.

CIELAB is a colorimetric system that numerically replaces colors with a color space close to what humans perceive $(20,21)$. It is useful for comparing visibility in endoscopy. We found that, when using CIELAB color differences in $\mathrm{CS}$, linked-color imaging was useful for identifying $\mathrm{CP}$, 
Adenomatous polyps

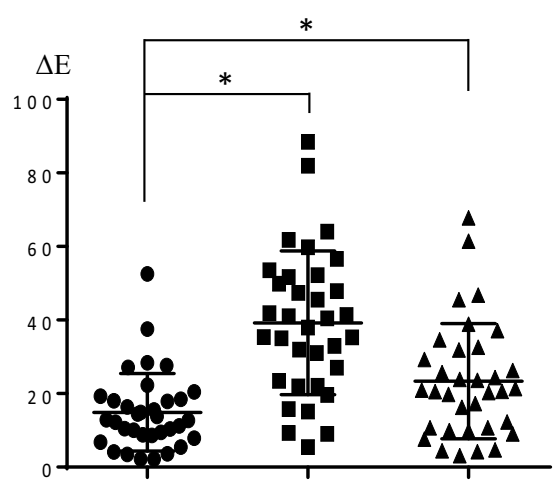

$\mathrm{WL} \Delta \mathrm{E} \quad \mathrm{FICE} \Delta \mathrm{E} \quad \mathrm{BM} \Delta \mathrm{E}$
Hyperplastic polyps

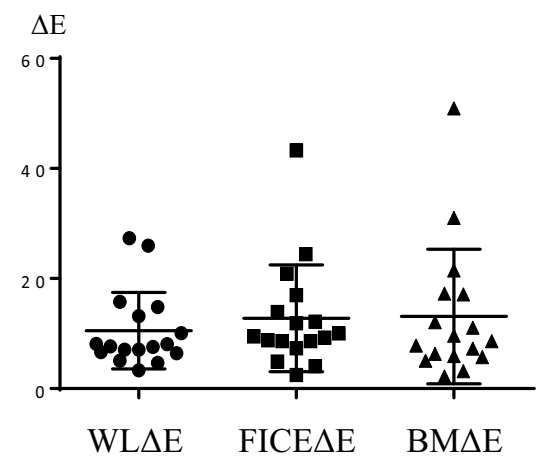

$* p<0.001$

Figure 4. Color differences in the colon capsule endoscopy (CCE) images of APs and HPs. The FICE $\Delta E$ and $B M \Delta E$ were significantly higher than the $W L \Delta E$ in $A P$ images $(p<0.001)$. In contrast, the FICE $\Delta E$ and $B M \Delta E$ were not significantly different from the WLAE in HP images ( $p=0.44$ and $\mathrm{p}=0.45$, respectively). WL: white light, FICE: flexible spectral imaging color enhancement, BM: blue mode, $\Delta \mathrm{E}$ : color differences

Table 3. The Ratio of the Color Differences in the CCE Images of Adenomatous and Hyperplastic Polyps.

\begin{tabular}{lccc}
\hline & Adenomatous polyps & Hyperplastic polyps & $\mathrm{p}$ value \\
\hline FICE $\Delta \mathrm{E}^{\prime}$ & $3.3 \pm 1.8$ & $1.3 \pm 0.6$ & $<0.001$ \\
$\mathrm{BM} \Delta \mathrm{E}^{\prime}$ & $1.6 \pm 0.5$ & $1.2 \pm 0.6$ & 0.227 \\
\hline
\end{tabular}

CCE: colon capsule endoscopy, FICE: flexible spectral imaging color enhancement, $\mathrm{BM}$ : blue mode, $\Delta \mathrm{E}^{\prime}$ : the ratio of the color differences

and blue-laser imaging was useful when observing magnified images (21). Furthermore, it was reported that, when using this method with SBCE, FICE was effective in improving the detection rate of small bowel lesions (18). In the present study, we showed that using the CIELAB color difference in FICE images from CCE to calculate the $\triangle \mathrm{E}^{\prime}$ and determine a cut-off value enabled the differentiation of APs from HPs with high sensitivity and specificity. While it was previously reported that using narrow-band imaging improves the ability to differentiate between AP and HP with CS (41-43), this is the first report on CCE. Sato et al. reported that visibility is improved to a greater degree with FICE than with BM, especially for vascular lesions, such as angioectasia, to emphasize the redness of the lesions (18). As APs appear redder than HPs, FICE $\Delta$ E' but not $\mathrm{BM} \Delta \mathrm{E}$ ' can differentiate between APs and HPs with high accuracy. The FICE used in the present study was installed in the CCE system (Covidien Japan), and no new specialized equipment was required. Therefore, this method can be widely applied and is expected to be of great use.

Several limitations associated with the present study warrant mention. First, we only examined APs and HPs, excluding malignant tumors, SSA/Ps, and other lesions. Nevertheless, the value of differentiating with FICE $\Delta \mathrm{E}$ ' is its ability to reduce unnecessary CS. We believe that it is highly likely

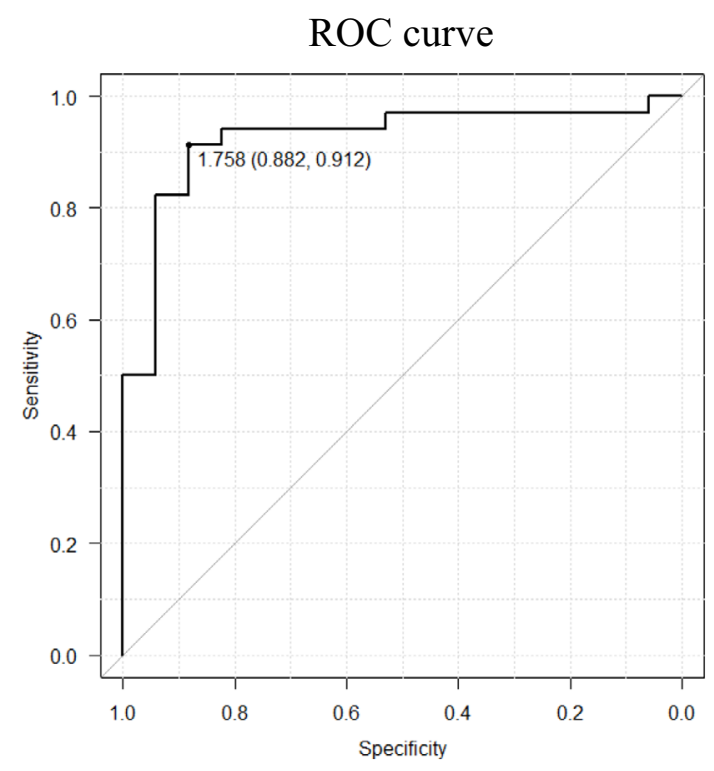

Figure 5. Receiver operating characteristic (ROC) curve for FICE $\Delta E$ ' in the CCE images of adenomatous vs. hyperplastic polyps. The area under the curve (AUC) was 0.928 (95\% confidence interval $0.843-1$ ). The optimal cut-off value that allows for the differentiation of these polyps was 1.758 . This cut-off value had $91.2 \%$ sensitivity and $88.2 \%$ specificity.

that there is no need to rush to perform CS for polyps that have a FICE $\Delta E^{\prime} \leq 1.758$ and are $<10 \mathrm{~mm}$. Second, even if APs and HPs can be differentiated with high sensitivity and specificity using FICE $\Delta$ E', a histopathological diagnosis by a biopsy is not possible with CCE; thus, a definitive diagnosis cannot be made. Third, while we examined cases in which the polyps observed with CCE were confirmed with $\mathrm{CS}$, there was no guarantee that these were the same polyps. In the present study, we only examined polyps that were in 
the same location or adjacent areas with CCE and CS, that did not differ in size, and that were confirmed by an interpreter to be the same polyp. We therefore believe they were almost always the same polyps. Fourth, this was a retrospective, single-center study, and the sample size was small. A prospective multicenter trial in a larger population should be performed to confirm the clinical usefulness of this method.

In conclusion, APs and HPs can be differentiated with high accuracy by using FICE on CCE images and applying a CIELAB color difference analysis. This method has the potential to reduce unnecessary CS procedures. To clarify the clinical usefulness of this method, a multicenter trial would be worth performing in a future study.

\section{The authors state that they have no Conflict of Interest (COI).}

Kei Nakazawa and Sadaharu Nouda contributed equally to this work.

\section{References}

1. Ferlay J, Soerjomataram I, Dikshit R, et al. Cancer incidence and mortality worldwide: sources, methods and major patterns in GLOBOCAN 2012. Int J Cancer 136: E359-E386, 2015.

2. Torre LA, Bray F, Siegel RL, Ferlay J, Lortet-Tieulent J, Jemal A. Global cancer statistics, 2012. CA Cancer J Clin 65: 87-108, 2015.

3. Bond JH. Clinical evidence for the adenoma-carcinoma sequence, and the management of patients with colorectal adenomas. Semin Gastrointest Dis 11: 176-184, 2000.

4. Mandel JS, Bond JH, Church TR, et al. Reducing mortality from colorectal cancer by screening for fecal occult blood. Minnesota Colon Cancer Control Study. N Engl J Med 328: 1365-1371, 1993.

5. Mandel JS, Church TR, Ederer F, Bond JH. Colorectal cancer mortality: effectiveness of biennial screening for fecal occult blood. J Natl Cancer Inst 91: 434-437, 1999.

6. Eliakim R, Fireman Z, Gralnek IM, et al. Evaluation of the PillCam Colon capsule in the detection of colonic pathology: results of the first multicenter, prospective, comparative study. Endoscopy 38: 963-970, 2006.

7. Eliakim R, Yassin K, Niv Y, et al. Prospective multicenter performance evaluation of the second-generation colon capsule compared with colonoscopy. Endoscopy 41: 1026-1031, 2009.

8. Spada C, Hassan C, Munoz-Navas M, et al. Second-generation colon capsule endoscopy compared with colonoscopy. Gastrointest Endosc 74: 581-589. e1, 2011.

9. Saito Y, Saito S, Oka S, et al. Evaluation of the clinical efficacy of colon capsule endoscopy in the detection of lesions of the colon: prospective, multicenter, open study. Gastrointest Endosc 82: 861869, 2015.

10. Van Gossum A, Munoz-Navas M, Fernandez-Urien I, et al. Capsule endoscopy versus colonoscopy for the detection of polyps and cancer. N Engl J Med 361: 264-270, 2009.

11. Busegeanu C FA, Stemate A, Negreanu L. A series of images of digestive cancers using Pill Cam COLON2 video capsule endoscopy. J Med Life 7: 529-532, 2014.

12. Muguruma N, Tanaka K, Teramae S, Takayama T. Colon capsule endoscopy: toward the future. Clin J Gastroenterol 10: 1-6, 2017.

13. Pohl J, May A, Rabenstein T, Pech O, Ell C. Computed virtual chromoendoscopy: a new tool for enhancing tissue surface structures. Endoscopy 39: 80-83, 2007.
14. Fedeli P, Gasbarrini A, Cammarota G. Spectral endoscopic imaging: the multiband system for enhancing the endoscopic surface visualization. J Clin Gastroenterol 45: 6-15, 2011.

15. Togashi K, Osawa H, Koinuma K, et al. A comparison of conventional endoscopy, chromoendoscopy, and the optimal-band imaging system for the differentiation of neoplastic and non-neoplastic colonic polyps. Gastrointest Endosc 69: 734-741, 2009.

16. Abdelaal UM, Morita E, Nouda S, et al. Blue mode imaging may improve the detection and visualization of small-bowel lesions: a capsule endoscopy study. Saudi J Gastroenterol 21: 418-422, 2015.

17. Imagawa $H$, Oka $S$, Tanaka $S$, et al. Improved visibility of lesions of the small intestine via capsule endoscopy with computed virtual chromoendoscopy. Gastrointest Endosc 73: 299-306, 2011.

18. Sato Y, Sagawa T, Hirakawa M, et al. Clinical utility of capsule endoscopy with flexible spectral imaging color enhancement for diagnosis of small bowel lesions. Endosc Int Open 2: E80-E87, 2014.

19. Krystallis C, Koulaouzidis A, Douglas S, Plevris JN. Chromoendoscopy in small bowel capsule endoscopy: Blue mode or Fuji Intelligent Colour Enhancement? Dig Liver Dis 43: 953-957, 2011.

20. Kuehni RG. Color-tolerance data and the tentative CIE 1976 L*a*b* formula. J Opt Soc Am 66: 497-500, 1976.

21. Tanaka Y, Inoue T, Kakimoto K, et al. Evaluation of the impact of linked color imaging for improving the visibility of colonic polyp. Oncol Lett 18: 5555-5560, 2019.

22. Rex DK, Adler SN, Aisenberg J, et al. Accuracy of capsule colonoscopy in detecting colorectal polyps in a screening population. Gastroenterology 148: 948-957. e2, 2015.

23. Spada C, Hassan C, Barbaro B, et al. Colon capsule versus CT colonography in patients with incomplete colonoscopy: a prospective, comparative trial. Gut 64: 272-281, 2015.

24. Participants in the Paris Workshop. The Paris endoscopic classification of superficial neoplastic lesions: esophagus, stomach, and colon. Gastrointest Endosc 58: 3-43, 2003.

25. Vogelstein B, Fearon ER, Hamilton SR, et al. Genetic alterations during colorectal-tumor development. N Engl J Med 319: 525$532,1988$.

26. Fearon ER, Vogelstein B. A genetic model for colorectal tumorigenesis. Cell 61: 759-767, 1990.

27. Laiyemo AO, Murphy G, Sansbury LB, et al. Hyperplastic polyps and the risk of adenoma recurrence in the polyp prevention trial. Clin Gastroenterol Hepatol 7: 192-197, 2009.

28. Kashida H, Kudo SE. Early colorectal cancer: concept, diagnosis, and management. Int J Clin Oncol 11: 1-8, 2006.

29. Aldridge AJ, Simson JN. Histological assessment of colorectal adenomas by size. Are polyps less than $10 \mathrm{~mm}$ in size clinically important? Eur J Surg 167: 777-781, 2001.

30. Ahlawat SK, Gupta N, Benjamin SB, Al-Kawas FH. Large colorectal polyps: endoscopic management and rate of malignancy: does size matter? J Clin Gastroenterol 45: 347-354, 2011.

31. Ponugoti PL, Cummings OW, Rex DK. Risk of cancer in small and diminutive colorectal polyps. Dig Liver Dis 49: 34-37, 2017.

32. Gupta N, Bansal A, Rao D, et al. Prevalence of advanced histological features in diminutive and small colon polyps. Gastrointest Endosc 75: 1022-1030, 2012.

33. Lieberman DA, Rex DK, Winawer SJ, Giardiello FM, Johnson DA, Levin TR. Guidelines for colonoscopy surveillance after screening and polypectomy: a consensus update by the US MultiSociety Task Force on Colorectal Cancer. Gastroenterology 143: 844-857, 2012.

34. Ferlitsch M, Moss A, Hassan C, et al. Colorectal polypectomy and endoscopic mucosal resection (EMR): European Society of Gastrointestinal Endoscopy (ESGE) Clinical Guideline. Endoscopy 49: 270-297, 2017.

35. Rex DK, Ahnen DJ, Baron JA, et al. Serrated lesions of the col- 
orectum: review and recommendations from an expert panel. Am J Gastroenterol 107: 1315-1329, 2012.

36. Leggett B, Whitehall V. Role of the serrated pathway in colorectal cancer pathogenesis. Gastroenterology 138: 2088-2100, 2010.

37. Lash RH, Genta RM, Schuler CM. Sessile serrated adenomas: prevalence of dysplasia and carcinoma in 2139 patients. J Clin Pathol 63: 681-686, 2010.

38. Owens SR, Chiosea SI, Kuan SF. Selective expression of gastric mucin MUC6 in colonic sessile serrated adenoma but not in hyperplastic polyp aids in morphological diagnosis of serrated polyps. Mod Pathol 21: 660-669, 2008.

39. Matsumoto T, Mizuno M, Shimizu M, Manabe T, Iida M. Clinicopathological features of serrated adenoma of the colorectum: comparison with traditional adenoma. J Clin Pathol 52: 513-516, 1999.

40. Okamoto K, Kitamura S, Kimura $T$, et al. Clinicopathological characteristics of serrated polyps as precursors to colorectal cancer: current status and management. J Gastroenterol Hepatol 32: 358-367, 2017.
41. Machida H, Sano Y, Hamamoto Y, et al. Narrow-band imaging in the diagnosis of colorectal mucosal lesions: a pilot study. Endoscopy 36: 1094-1098, 2004.

42. Rastogi A, Keighley J, Singh V, et al. High accuracy of narrow band imaging without magnification for the real-time characterization of polyp histology and its comparison with high-definition white light colonoscopy: a prospective study. Am J Gastroenterol 104: 2422-2430, 2009.

43. Hewett DG, Kaltenbach T, Sano Y, et al. Validation of a simple classification system for endoscopic diagnosis of small colorectal polyps using narrow-band imaging. Gastroenterology 143: 599607, 2012.

The Internal Medicine is an Open Access journal distributed under the Creative Commons Attribution-NonCommercial-NoDerivatives 4.0 International License. To view the details of this license, please visit (https://creativecommons.org/licenses/ by-nc-nd/4.0/).

(C) 2021 The Japanese Society of Internal Medicine Intern Med 60: 1805-1812, 2021 